\title{
Distal femoral opening-wedge osteotomy for lateral compartment osteoarthritis of the knee
}

\author{
Dirk HPW Das' \\ Thea Sijbesma ${ }^{2}$ \\ Henk J Hoekstra ${ }^{2}$ \\ Willem M van Leeuwen ${ }^{2}$ \\ 'Department of Orthopaedics \\ and Traumatology, Máxima Medisch \\ Centrum Veldhoven, The Netherlands; \\ ${ }^{2}$ Department of Orthopaedic Surgery, \\ The St Anna Hospital, Geldrop, \\ The Netherlands
}

\begin{abstract}
We retrospectively evaluated 12 patients with lateral gonarthritis after a distal femoral lateral opening osteotomy using a Puddu-plate. Thirteen patients with lateral gonarthritis and genu valgum were operated upon. One patient died during follow-up. We used the Lysholm score and the adapted Hospital for Special Surgery (HSS) clinical and functional score. At 34 months follow up, all patients had a functional and clinical evaluation. All patients responded to a questionnaire over the telephone regarding the survival, Lysholm, and functional HSS score at 74 months follow-up. The average age at operation was 52 years. The average correction angle was $11^{\circ}\left(16^{\circ}\right.$ to $\left.5^{\circ}\right)$. At 34 months follow up, the functional HSS score improved from 58 to 72 points. At 74 months follow up, the Lysholm score improved from 64 to 77 points. The clinical horizon scanning system score improved from 42 points presurgery to 64 postsurgery. Two knees were converted to total knee arthroplasty due to persisting postoperative pain. Lateral supracondylar opening-wedge osteotomy is a satisfying treatment for lateral osteoarthritis of the knee with genu valgum in younger patients.

Keywords: lateral gonarthritis, femoral osteotomy, Lysholm score, knee
\end{abstract}

\section{Introduction}

The knee is the most common site of osteoarthritis (OA) after the hip. Isolated, lateralcompartment arthritis is frequently associated with a valgus deviation of the knee. The valgus deformity increases the impact of weight bearing on the lateral compartment and may deteriorate the arthritis. The angulation will deteriorate and result in an incongruent knee joint.

Treatment options for osteoarthritis of the knee are: bracing, medication, arthroscopic debridement, osteotomy, unicompartmental arthroplasty and total knee arthroplasty (TKA) (Iorio and Healy 2003). Correction of the mechanical axis of the leg by means of an osteotomy might reduce or stop the progression of lateral gonarthritis and postpone or prevent TKA. Osteotomy of the femur in genu valgum has also the advantage that a total knee arthroplasty performed in the future is easier to perform due to a better alignment.

In this study we retrospectively evaluated the clinical and functional results of distal femoral opening wedge osteotomy in the treatment of lateral osteoarthritis with genu valgum.

\section{Materials and methods}

Between 1997 and 2002, 13 distal femoral opening wedge osteotomies were performed according to Puddu (Arthrex $\left.{ }^{\circledR}\right)$ at the distal epiphyseal region. All patients are included in this study. Patients with a revision of the osteotomy to a TKA are considered as a failure and were evaluated according their lowest scores. During follow-up one patient died of causes unrelated to the knee surgery. The remaining 12 patients were seen clinically at an average of 34 (11-49) months after surgery and were contacted by 
telephone after a mean 74 (51-89) months. The indication for an opening wedge osteotomy was mild to moderate lateral radiographic $\mathrm{OA}$, associated with genu valgum malalignment of more than $10^{\circ}$ in a patient with a biological age of less than 65 years. The average age at surgery was 55 years (46-71). We operated on 7 female and 5 male patients. Eight patients had previous lateral meniscectomy, two patients had a postfracture deformity. Contraindications for operation were collateral ligament laxity of the knee of more than $5 \mathrm{~mm}$, extension deficit of more than $5^{\circ}$, patella femoral OA, advanced medial OA, other major disabling physical conditions and prior major knee surgery.

Before surgery standard AP and lateral X-rays were made on large X-ray plates. The anatomical axis was assessed as the angle between the mid-shaft femur and mid-shaft tibia. The aim was to slightly overcorrect the anatomical axis to approximately $3^{\circ}$ of valgus (instead of the normal $5^{\circ}$ to $6^{\circ}$ ).

Four patients had a radiographic stage I OA of the lateral compartment according to Kellgren-Lawrence (joint space narrowing), 5 patients had stage II (joint space narrowing and osteophytes) and 3 patients had stage III OA (joint space narrowing, osteophytes and subchondral sclerosis). At the medial compartment, 3 patients had stage 0 (no signs of OA), 6 patients had stage I and 3 patients had stage II OA (See also Table 1).

The distal femur was approached laterally at the distal epiphyseal region. A 3-mm guide wire is drilled in the lateral condyl $1 \mathrm{~cm}$ superior to the lateral epicondyle, and directed to the origin of the medial collateral ligament (adductor tubercle) under fluoroscopic control. Over this wire a special sleeve is placed to determine the osteotomy plane. The osteotomy is made using an oscillating saw ending approximally $5 \mathrm{~mm}$ lateral of the medial cortex. The osteotomy plane is spread using special spreading wedges. The Puddu plate, which is designed with varying spacerblocks, is then placed over the osteotomy gap and secured proximally with traditional bicortical screws and distally with partially threaded cancellous screws. The wedge is filled with bone allografts (Figure 1). The patients were mobilized with partial weight bearing with 2 crutches and a splint for 8 weeks postoperative until radiological signs of healing were seen.

To evaluate the functional outcome, we used the Lysholm score (0-100) and the adapted Hospital for Special Surgery (HSS) clinical score (0-100). The functional evaluation was assessed with the functional HSS score (0-100) (Scott 1984). We divided the patients in excellent, good, fair, and poor as indicated by the scoring system (Table 2). The correlation between the degree of correction and results was assessed using Karl Pearson Product-Moment Correlation Coefficient. We also wanted to know the value of the preoperative OA op standard X-ray as a predictor of postoperative results.

\section{Results}

The average anatomical valgus correction was $11^{\circ}$, from an average preoperative valgus of $16^{\circ}\left(10^{\circ}-21^{\circ}\right)$ to a postoperative $5^{\circ}\left(1^{\circ}-8^{\circ}\right)$.

At 34 months follow up the average Lysholm score improved from 64 points to 76 points, the clinical HSS knee score improved from 42 points to 64 points and the functional HSS score improved form 58 to 67 . After 74 months follow up we reassessed the Lysholm and functional HSS score. There was only a slight improvement of the HSS score to 72 points (Table 3 ).

In 7 patients the Puddu plate was removed at an average of 25 months postsurgery because of irritation at the iliotibial tract. Patients mentioned subjectively that removal of the Puddu plate improved the iliotibial tract irritation, however this could not be seen in the scoring results because no separate data before and after removal of the plate was assessed.

In Table 2 we grouped the patients according to their score in excellent, good, fair, and poor as indicated. In

Table I Preoperative radiographic OA staging according to Kellgren-Lawrence (K-L) in relation to the postoperative change in outcome

\begin{tabular}{|c|c|c|c|c|c|c|c|c|}
\hline \multicolumn{5}{|c|}{ Medical compartment } & \multicolumn{4}{|c|}{ Lateral compartment } \\
\hline K-L Grade & $\mathbf{n}=$ & $\Delta$ Lysholm & $\Delta$ Clinical HSS & $\Delta$ Functional HSS & $\mathbf{n}=$ & $\Delta$ Lysholm & $\Delta$ Clinical HSS & $\Delta$ Functional HSS \\
\hline 0 & 3 & +21 & +29 & +23 & - & & & \\
\hline I & 6 & +25 & +32 & +24 & 4 & +27 & +33 & +20 \\
\hline II & 3 & -24 & -4 & -23 & 5 & 0 & +20 & +3 \\
\hline III & - & & & & 3 & +29 & +10 & +50 \\
\hline IV & - & & & & - & & & \\
\hline
\end{tabular}

Abbreviation: HSS, Hospital for Special Surgery; OA, osteoarthritis. 


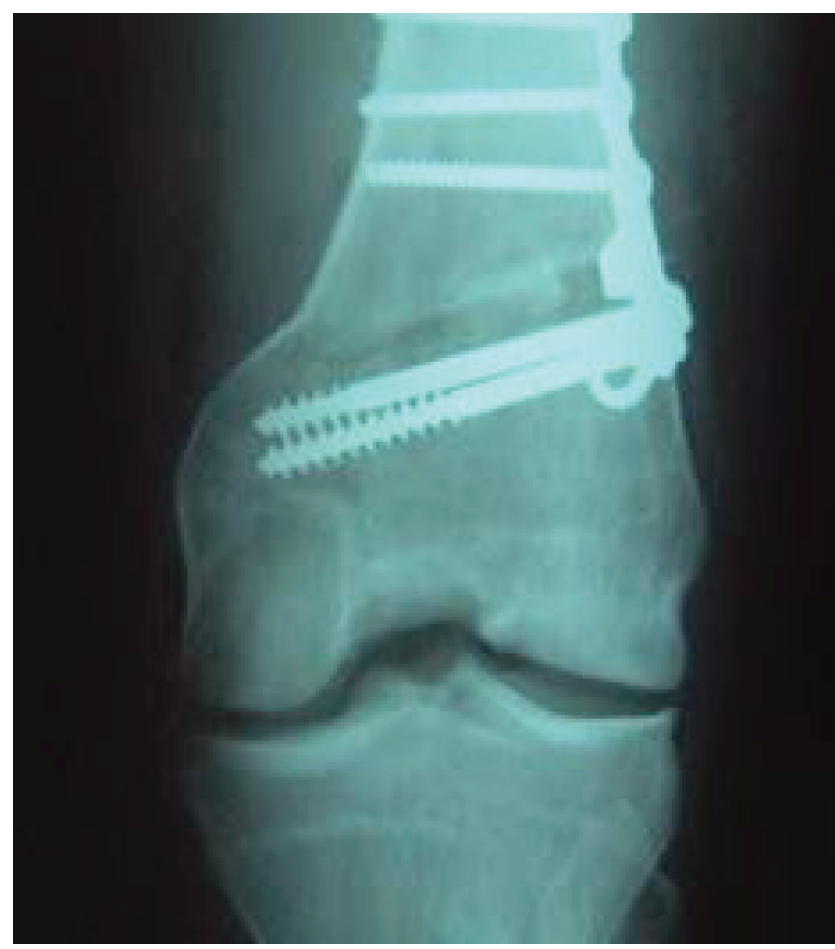

Figure I Distal femoral opening wedge osteotomy in lateral gonarthritis.

respect to all three scoring subjects the number of patients rated as a failure (fair or poor) has decreased after 74 months compared to 34 months follow-up. However, at follow up not all patients are satisfied. Three patients have deteriorated postsurgery and were in fact set back by the osteotomy. Two of those had a TKA at 37 and 42 months postosteotomy, for persisting pain even after plate removal. These two patients had the most advanced lateral $\mathrm{OA}$ of the group on preoperative X-rays. Both patients had lateral stage III and medial II and I, respectively. In retrospective, we assume that the advanced OA on the medial side was still causing the postoperative pain. Those 2 patients should have been offered a TKA in the first place. In Table 1 and 2 those 2 patients are included in the final follow up and graded according to their last known results before TKA.
The third patient had a pronounced preoperative valgus deformity of $21^{\circ}$ due to a mal-union of a condylar fracture in childhood. Although she hardly experienced any pain, we decided to proceed with an osteotomy, hoping to stop progression of a beginning radiographic arthritic process, and if not successful to diminish the technical hazard of an eventual TKA. After the surgery she persisted to have severe and unexplained pain in the back of the knee. She is now scheduled to have plate and screws removed.

Patients with advanced medial OA preoperative had less functional improvement and a lower Lysholm score at follow up However, patients with K-L stage III OA at the lateral side of the knee improved in all scoring results (Table 1).

We assessed the correlation between the correction angel and the 74 months postoperative result using the Karl Pearson coefficient and see that the correlation is negative in the functional HSS and Lysholm score $(-0.68$ and -0.61 , respectively) and is nil in the clinical HSS score (0.01). This means that patients with major correction had inferior functional results.

One patient had a delayed union resulting in a 7-month rehabilitation period. No cases of infection, neurovascular problems, or broken Puddu plate occurred during the postoperative period.

\section{Discussion}

Osseous correction of genu valgum can theoretically be done at two levels and four surgical options are available: medial closing wedge osteotomy above or below the knee joint, and lateral opening wedge osteotomy above or below the knee joint. As a rule one should always correct at the site of the deformity. In a valgus gonarthritis, the deformity is most often located in the distal femur and should be corrected over there. Lateral opening osteotomies theoretically elongate the peroneal nerve at the level of a tight trajectory around the fibular head. However in our series there were no cases of peroneal damage. Most studies evaluating distal medial femoral closing wedge osteotomies, showed its value in

Table 2 Clinical and functional scores. Classifications in excellent, good, fair, and poor

\begin{tabular}{|c|c|c|c|c|c|c|c|c|c|}
\hline \multirow[b]{3}{*}{ Excellent } & \multicolumn{3}{|c|}{ Lysholm } & \multicolumn{3}{|c|}{ Clinical knee score HSS } & \multicolumn{3}{|c|}{ Functional knee score HSS } \\
\hline & \multicolumn{2}{|c|}{ Presurgery } & \multirow{2}{*}{$\begin{array}{l}74 \text { months } \\
2\end{array}$} & \multicolumn{2}{|l|}{ Presurgery } & \multirow{2}{*}{$\begin{array}{l}34 \text { months } \\
2\end{array}$} & \multicolumn{2}{|l|}{ Presurgery } & \multirow{2}{*}{$\begin{array}{l}74 \text { months } \\
2\end{array}$} \\
\hline & 1 & $(95-100)$ & & - & $(85-100)$ & & 2 & $(85-100)$ & \\
\hline Good & - & $(84-94)$ & 5 & 2 & $(70-84)$ & 2 & 3 & $(70-84)$ & 5 \\
\hline Fair & 2 & $(65-83)$ & - & - & $(60-69)$ & 2 & - & $(60-69)$ & - \\
\hline Poor & 9 & $(<64)$ & 5 & 10 & $(<60)$ & 6 & 7 & $(<60)$ & 5 \\
\hline
\end{tabular}

Abbreviation: HSS, Hospital for Special Surgery. 
Table 3 Average preoperative and postoperative scores

\begin{tabular}{llll}
\hline & Presurgery & $\mathbf{3 4}$ months & $\mathbf{7 4}$ months \\
\hline Lysholm score & 64 & 76 & 77 \\
Clinical knee score HSS & 42 & 64 & - \\
Functional knee score HSS & 58 & 67 & 72 \\
\hline
\end{tabular}

Abbreviation: HSS, Hospital for Special Surgery.

painful genu valgum (Healy et al 1988; McDermitt et al 1988; Terry and Cimino 1992; Edgertin et al 1993; Finkelstein et al 1996; Mathews et al 1998; Philips and Krakow 1999; Agligetti and Menchetti 2000). Our results were comparable to those given in literature. Cameron and colleagues (1997) evaluated 49 medial closing-wedge osteotomies of the distal femur. They found no correlation between sex, age, or preoperative valgus angle and postoperative outcome. Osteotomy appeared inferior to TKA in pain reduction. However, we believe that osteotomy should not be seen as an alternative to TKA but rather as an option to prevent or postpone it, at younger age.

Distal femoral opening wedge osteotomy has some interesting advantages compared to the other osteotomies, which made us decide for open wedge osteotomy. Lateral access to the distal femur is easy and the chance of peroneal damage is limited (Healy and Barber 1990) compared to a lateral osteotomy at the level of the proximal tibia. An opening wedge osteotomy elongates the malaligned leg, which is often a little shorter. Only one saw cut needs to be made, which reduces the possibility of preoperative complications. Knee replacement after distal femoral osteotomy can offer difficulties in assessing the mechanical axis. Careful preoperative templating for creating the entrance hole for intramedullary guidance or extramedullary femoral alignment might be needed. TKA after an osteotomy also has a higher infection risk than a primary TKA (Cameron and Park 1997; Papadopoulos et al 2002; Nelson et al 2003). The two patients in our series that needed TKA had an uneventful replacement with satisfying pain relief and good range of motion.

This study has several shortcomings. As with all retrospective studies, we lack uniformity in data collection and radiographic documentation. We do not have standing whole leg radiological films pre- and postoperative. The radiology department in our hospital was not able at that time to produce such pictures. As a result of this, the measured correction is not highly reproducible and is, at best, an indication of the real alignment. Nowadays this has changed, and we do not perform an osteotomy without whole leg films anymore.
Large prospective randomized trials evaluating the results of lateral opening wedge osteotomies are not available so far.

This study of a small cohort with relative short follow up and no other available large studies on this subject, do not permit us to make strong recommendations in favor of lateral opening-wedge osteotomy in the treatment of lateral OA with genu valgum. However, it appears an attractive technique to relief pain in patients with lateral OA and genu valgum at younger age.

Before considering an osteotomy one must realize that consecutive operations might be needed (osteotomy and plate removal) all with their associated complication risks. Therefore we can not state that an osteotomy is a successful alternative for TKA. Individual judgment is always needed to decide between the osteotomy and the TKA.

\section{Conclusion}

Lateral femoral opening-wedge osteotomy offers pain relief and anatomical correction in patients with painful lateral OA associated with genu valgum at younger age. However some patients still experience pain mainly because of advanced medial osteoarthritis. The Puddu technique and plate fixation offer satisfactory stability for early rehabilitation. Adequate patient selection is the keystone for a successful outcome. Patients with advanced OA or major angulation had unsuccessful results in our study.

\section{Disclosure}

No funds of any kind were received for this research.

\section{References}

Aglietti P, Menchetti PPM. 2000. Distal femoral varus osteotomy in the valgus osteoarthritic knee. Am J Knee Surg, 13:89-95.

Cameron HU, Botsford DJ, Park YS. 1997. Prognostic factors in the outcome of supracondylar femoral osteotomy for lateral compartment osteoarthritis of the knee. Can J Surg, 40:114-8.

Cameron HU, Park YS. 1997. Total knee replacement after supracondylar femoral osteotomy. Am J Knee Surg, 10:70-2.

Edgerton BC, Mariani EM, Morrey BF. 1993. Distal femoral varus osteotomy for painful genu valgum. Clin Orth, 288:263-9.

Finkelstein JA, Gross AE, Davis A. 1996. Varus osteotomy of the distal part of the femur. J Bone Joint Surg, 78-A:1348-52.

Healy WJ, Barber TC. 1990. The role of osteotomy in the treatment of osteoarthritis of the knee. Am J Knee Surg, 3:79-109. 
Healy WL, Anglen JO, Wasilewski SA, et al. 1988. Distal femoral varus osteotomy. J Bone Joint Surg, 70-A:102-9.

Iorio R, Healy WL. 2003. Current concepts review; Unicompartmental arthritis of the knee. J Bone Joint Surg, 85-A:1351-64.

Mathews J, Cobb AG, Richardson S, et al. 1998. Distal femoral osteotomy for lateral compartment osteoarthritis of the knee. Orthopedics, 21:437-40.

McDermott PAG, Finklestein JA, Rarine I, et al. 1988. Distal femoral varus osteotomy for valgus deformity of the knee. J Bone Joint Surg, 70-A:110-16.

Nelson CL, Saleh KJ, Kassim RA, et al. 2003. Total knee arthroplasty after varus osteotomy of the distal part of the femur. J Bone Joint Surg, 85-A:1062-5.
Papadopoulos EC, Parvizi J, Lai CH, et al. 2002. Total knee arthorplasty following prior distal femoral fracture. Knee, 9:267-74.

Philips MJ, Krackow KA. 1999. High tibial osteotomy and distal femoral osteotomy for valgus or varus deformity around the knee. Instr Course Lect, 48:125-9.

Scott WN. 1984. Insall and Scott Surgery of the Knee, 3th ed. New York: Churchill Livingstone, pp. 975-82

Terry GC, Cimino PM. 1992. Distal femoral osteotomy for valgus deformity of the knee. Orthopedics, 15:1283-90. 
